\title{
Properties of High $\beta$, Quasi-Axisymmetric NCSX Stellarator Configurations
}

\author{
L. P. Ku, G. Y. Fu, D. Monticello, A. Reiman \\ Princeton Plasma Physics Laboratory \\ Princeton, NJ 08543
}

\author{
A. Boozer \\ Columbia University \\ New, York, NY 10027
}

\begin{abstract}
Quasi-axisymmetry, external kinks and ballooning stability are studied with respect to the plasma shaping and the variation in the pressure and current profiles for NCSX. We show that while the kink stability may require a delicate boundary shaping, most quasiaxisymmetry may be achieved using a few low order modes that eliminate the large mirror fields arising partly from boundary shaping for the kink stability. In addition, we demonstrate that the kink and ballooning instability may be improved in the NCSX configurations by a more peaked pressure profile or a broader current profile. Finally, we show numerically that it is possible to construct a quasi-axisymmetric configuration that is stable to the external kink at all current levels for which the edge rotational transform is less than 0.5 .
\end{abstract}

\section{INTRODUCTION}

Configurations having good quasi-axisymmetry (QA) and favorable stability properties were developed recently for the National Compact Stellarator Experiment (NCSX) [1,2]. These are three-field periods devices typically with major radii $\sim 1.45 \mathrm{~m}$, aspect ratios $(\mathrm{R} /<\mathrm{a}>) \sim 3.5$, and stability $\beta \sim 4 \%$. A substantial fraction of the rotational transform is internally generated by the bootstrap current expected to arise in the near two-dimensional magnetic structure. The overall rotational transform is made to be monotonically increasing outward by means of an external transform having a large positive shear.

In addition, these configurations are characterized by a strong shaping of the underlying axisymmetric components to achieve good ballooning stability. This axisymmetric part of the shaping and the pressure and current profiles are taken from those of the ARIES reversed-shear tokamak studies [3]. Our configurations are further characterized by a strong non-axisymmetric shaping optimized to achieve the kink stability without a conducting wall. The configurations so obtained turned out to be stable also to the periodicity preserving vertical modes $[4,5]$. It is interesting to note that, unlike most of the Helias devices recently developed, our configurations have an outboard indentation at the half-period cross section. This indentation has been identified to be 
strongly associated with the kink stability. Also, the magnetic axis of these configurations is essentially circular and planer.

The procedures we used to optimize the configurations have been discussed in detail in Ref. [2]. The process was to satisfy the demand of kink stability and QA, subject to the constraints of the rotational transform being monotonically increasing, physical location being within maximum and minimum allowables, and in some instances, the coil current density being low. The Fourier harmonics describing the plasma boundary were altered in a complex way. To help understand the interplay, we re-built one of the configurations with gradually increasing number of boundary modes. The kink stability and QA were examined at each stage. In section II, we present results of this anatomical study.

The configurations obtained from the optimization were based on a set of reference pressure and current profiles. These profiles were consistent with the bootstrap current and chosen to provide optimal performance for a tokamak, based upon which our configurations were derived [2]. In section III, we present a sensitivity study showing the effects of pressure and current profile changes on the QA and stability properties.

Finally, in section IV, we show numerically that there exists a narrow stability gap for the external kinks at an edge rotational transform just below 0.5. We illustrate a new configuration that is more robust to plasma current excursion based on this observation.

The studies presented here provide us with important insight and yield useful data for our efforts in coil designs and in the preparation of plasma operations. We use QAS3_C82 shown in Ref. [2] as the primary example throughout the discussion.

\section{BOUNDARY SHAPE, QA AND KINK STABILITY}

We represent the last closed flux surface by an exponential series [6]:

$$
R+i Z=e^{i u} \sum \Delta_{m, n} e^{-i m u+i n v}
$$

where $R, Z$, and $\phi=v / \mathrm{N}_{\mathrm{fp}}$ are cylindrical coordinates about the principal axis of the stellarator, $\mathrm{N}_{\mathrm{fp}}$ is the number of field periods and $\mathrm{m}, \mathrm{n}$ are the poloidal and toroidal mode numbers, respectively. For QAS3_C82, there are $63 \Delta_{\mathrm{m}, \mathrm{n}}$, corresponding to five poloidal modes and three toroidal modes in the VMEC [7] representation that the optimization uses. We order these coefficients according to their magnitude weighted by the respective poloidal mode number (except for $\mathrm{m}=0$, in which case the weight is set to 1 ). Table 1 lists the most important $30 \Delta_{\mathrm{m}, \mathrm{n}}$.

Equilibria were generated using subsets of these coefficients, retaining anywhere from 6 modes to all. Fig. 1a shows the relative kink eigenvalue as a function of the number of modes retained, and Fig. $1 b$ shows the relative $\chi$ of the non-axisymmetric magnetic field spectrum in the Boozer coordinates, 
Table. $1 \Delta_{\mathrm{m}, \mathrm{n}}$ for QAS3_C82 ordered by $\left|\mathrm{m}^{*} \cdot \Delta_{\mathrm{m}, \mathrm{n}}\right|$, where $\mathrm{m}^{*}=\max \{1,|\mathrm{~m}|\}$. $\Delta_{0,0}$ is normalized to unity.

\begin{tabular}{crrrcrrr}
\hline Seq. No. & $\mathrm{m}$ & $\mathrm{n}$ & $\Delta_{\mathrm{m}, \mathrm{n}}$ & Seq. No. & $\mathrm{m}$ & $\mathrm{n}$ & $\Delta_{\mathrm{m}, \mathrm{n}}$ \\
\hline 1 & 1 & 0 & 3.098 & 16 & 4 & 0 & 0.010 \\
2 & 0 & 0 & 1.000 & 17 & 3 & 2 & 0.012 \\
3 & 2 & 0 & -0.310 & 18 & 4 & -1 & 0.009 \\
4 & 3 & 1 & 0.167 & 19 & -3 & -1 & 0.010 \\
5 & 2 & 1 & -0.176 & 20 & -4 & -2 & -0.006 \\
6 & 3 & 0 & 0.071 & 21 & 0 & 1 & 0.021 \\
7 & 4 & 2 & -0.044 & 22 & 0 & -1 & 0.021 \\
8 & 1 & 1 & -0.129 & 23 & 2 & -2 & 0.009 \\
9 & -1 & -1 & 0.107 & 24 & 0 & -2 & 0.017 \\
10 & -1 & 1 & 0.104 & 25 & 0 & 2 & 0.017 \\
11 & 4 & 1 & 0.026 & 26 & 2 & -1 & -0.009 \\
12 & -1 & 0 & 0.085 & 27 & 1 & -2 & 0.014 \\
13 & 2 & 2 & 0.026 & 28 & 4 & 3 & 0.003 \\
14 & 3 & -1 & -0.014 & 29 & -4 & -1 & 0.003 \\
15 & 1 & -1 & -0.041 & 30 & -3 & 0 & 0.004 \\
\hline
\end{tabular}

$$
\chi=\sqrt{\sum_{n \neq 0} B_{m, n}^{2}}
$$

The kink eigenvalues were calculated using the Terpsichore code [8].

We observe that with about half of the modes used, the kink stability and QA are already within a factor of 2 of the values of the final state. A plot of the cross sections of QAS3_C82 is shown in Fig. 2a. The fundamental structure may be described by the top six modes, which include the average major and minor radii, $\Delta_{1,0}, \Delta_{0,0}$; elongation, $\Delta_{2,0}$; triangularity, $\Delta_{3,0}$; and $l=2$ and $l=3$ stellarator components, $\Delta_{2,1}$ and $\Delta_{3,1}$. The rotational transform with these modes is quite close to that of the final configuration, but of course this simple configuration is neither kink stable nor QA. $\mathrm{B}_{0,1}$ is singularly the largest nonaxisymmetric magnetic field component which is $\sim 20 \%$ of $\mathrm{B}_{0,0}$ at $\mathrm{s}=0.5$ ( $\mathrm{s}$ being the toroidal flux label). Adding four more modes, primarily $\Delta_{4,2}$, an equilibrium that bears the signature of C82, namely, an indentation at the half-period cross section, is born. There is a significant reduction $(\sim 4)$ in the kink eigenvalue with little change in QA. The secondary mirror field, $\mathrm{B}_{0,2}$, becomes large, about $7 \%$ of $\mathrm{B}_{0,0}$. The primary mirror field, $\mathrm{B}_{0,1}$, remains at $\sim 17 \%$ level. Figs. $2 \mathrm{~b}$ and $2 \mathrm{c}$ show the cross sections of the last closed flux surface with the top six and ten modes retained.

Perhaps the most striking feature, examining Fig. 1, is that the kink eigenvalue reduces gradually, requiring ever more refined shaping, whereas the primary action for QA occurred just in the terms from 21 to 25 in Table 1 . The mirror fields, $\mathrm{B}_{0,1}$ and $\mathrm{B}_{0,2}$, 
still dominate the non-axisymmetry $(\sim 10 \%)$ when the top 20 modes are used to construct the equilibrium, with $\mathrm{B}_{1,2}, \mathrm{~B}_{1,-2}$ and $\mathrm{B}_{2,-2}$ about $1.5 \%$ each at $\mathrm{s}=0.5$. These low order components are effectively eliminated without compromising the kink stability by adding $\Delta_{0, \pm 1}, \Delta_{0, \pm 2}$ and $\Delta_{2,-2}$. With these addition, $\mathrm{B}_{0,2}$ is reduced to $0.7 \%$ and $\mathrm{B}_{0,1}$ to $1 \%$ at $\mathrm{s}=0.5$. Finally, when the top 30 modes are included in the construction of equilibrium, the magnitudes of the largest components, $\mathrm{B}_{2,1}$ and $\mathrm{B}_{1,2}$, are now just $0.8 \%$ at $\mathrm{s}=0.5$ due to the presence of $\Delta_{2,-1}$ and $\Delta_{1,-2}$. Also, the inclusion of $\Delta_{-4,-1}$ further reduces the kink eigenvalue by a factor $\sim 2$. Figs. $3 \mathrm{~d}$ and $3 \mathrm{e}$ show the cross-sections with the top 20 and 30 modes retained.

The decomposition of the optimized configuration serves useful purposes in the coil design and in the consideration of experimental flexibility. A main coil set may be designed to provide major shaping of the plasma (i.e. modes with large amplitude), while secondary coils may be designed to provide fine tuning tasks. The resulting coils may be simpler. In addition, the secondary coils may provide one with a useful knob to "tune" for different degrees of QA or kink growth rates. Several secondary coil sets may also be used to shape plasmas with somewhat different characteristics, hence increasing the flexibility of the experiment.
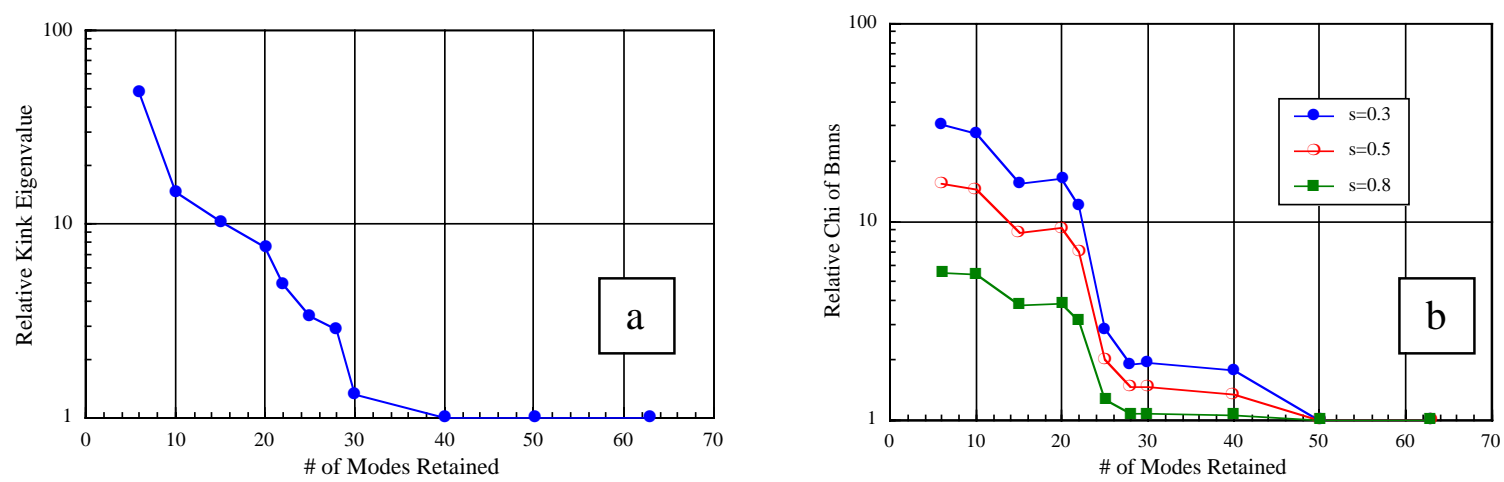

Fig. 1 (a) Relative kink eignenvalue, and (b) relative $\chi$ versus the retained number of boundary modes in QAS3_C82. $\chi$ is given for three surfaces: $s=0.3,0.5$ and 0.8 , where $s$ is the toroidal flux label. Lines are connected between calculated points to aid the eyes.

\section{PRESSURE/CURRENT PROFILES, STABILITY AND QA}

For the purpose of sensitivity studies, we allow pressure and current profiles to vary independently. We find that if the pressure profile becomes more peaked while the current profile is kept fixed, the stability properties are improved. To give an illustration, we prescribe the pressure profile, $p(s)$, as

$$
p(s)=p(0) \cdot\left(1.0-s^{\alpha_{1}}\right)^{\alpha_{2}}
$$



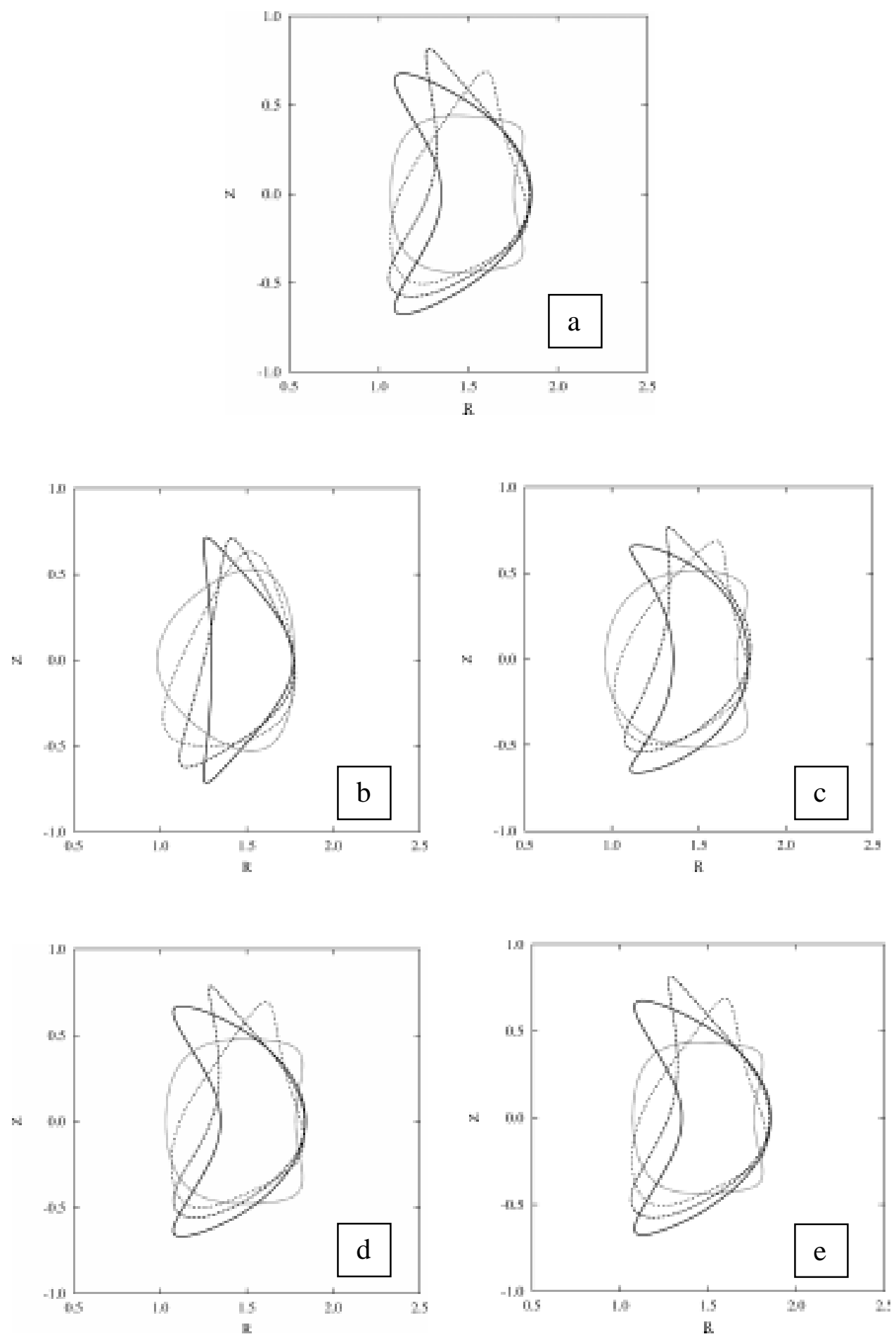

Fig. 2 Cross sections with partial boundary modes in QAS3_C82 used in equilibrium calculations: (a) all modes, (b) 6 modes, (c) 10 modes, (d) 20 modes, and (e) 30 modes. Cross sections are plotted at equal intervals over half a field period. 

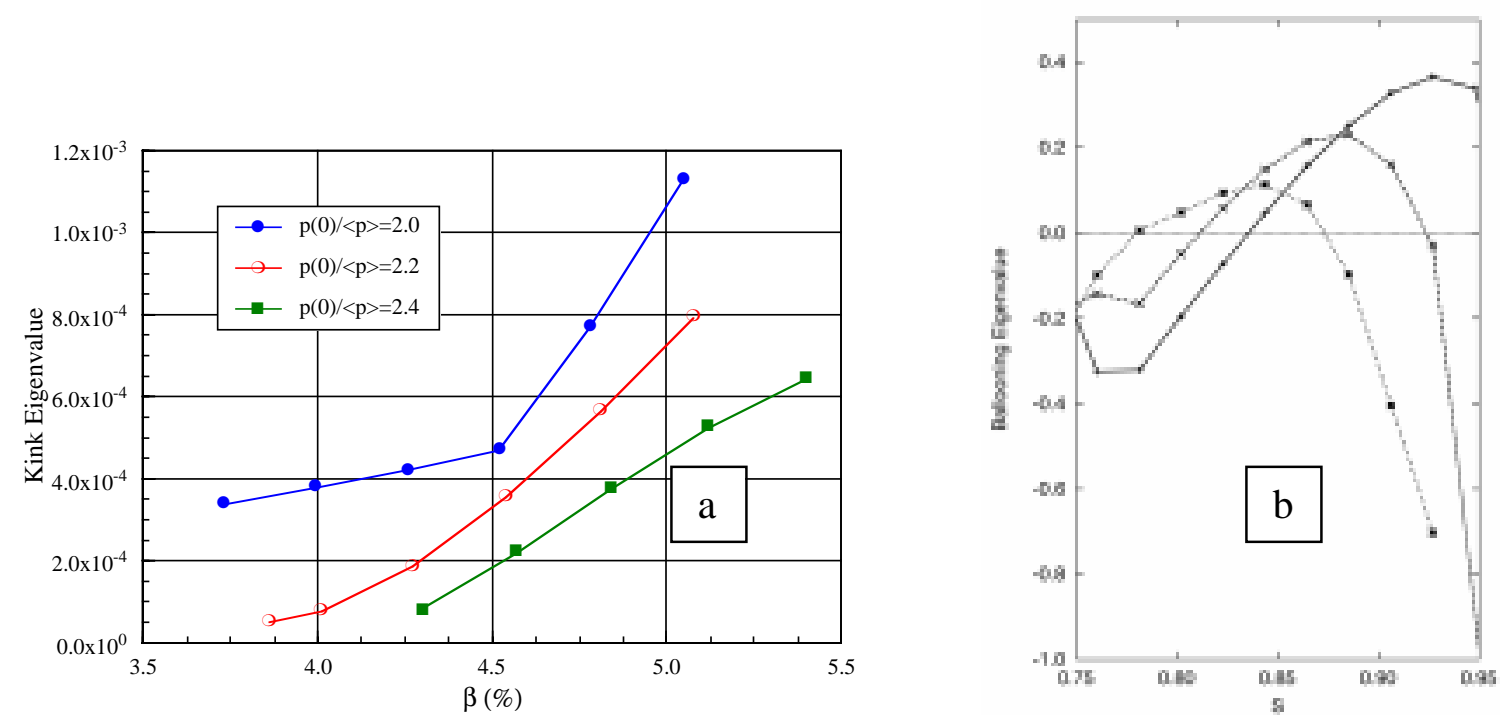

Fig. 3 (a) Kink eigenvalue versus $\beta$, and (b) ballooning eigenvalue at $4 \% \beta$ versus toroidal flux labal s, showing stability improvement as pressure profiles become increasingly peaked. Positive eigenvalue indicates instability. In (b), curve with solid line corresponds to $\mathrm{p}(0) /\langle\mathrm{p}\rangle=2.0$, dashed line 2.2 , and dotted line 2.4 .

For $\alpha_{1}=1.9$ and $\alpha_{2}=3.2$, QAS3_C82 is ballooning and kink stable at $4 \% \beta$ with the Aries current profile. For the study here, we use $\alpha_{1}=1.6$ and $\alpha_{2}=1.8,2.2$, and 2.6, which result in profiles with peaking factors, $\mathrm{p}(0) /\langle\mathrm{p}\rangle, 2.0,2.2$ and 2.4 , respectively. Fig. 3a shows the kink eigenvalue as a function of $\beta$ and Fig. $3 b$ shows the ballooning eigenvalue as a function of $\mathrm{s}$ at $4 \% \beta$ for these three profiles. The ballooning stability is calculated using the VVBAL code [9].

In our configurations, there does not exist a vacuum magnetic well (with the same boundary as that at full current and full $\beta$ ). When the external rotational transform ( 1 ) is increased to compensate for the decreasing internal $l$ beyond the shear reversal point (at $\mathrm{s} \sim 0.75$ ), the magnetic well generated by the finite pressure and current is weakened or even lost locally. The Mercier and ballooning modes tend to become unstable unless there is a large enough magnetic shear or low enough $\mathrm{p}^{\prime}$. For more peaked pressure profiles, $\mathrm{p}^{\prime}$ is smaller in the ballooning unstable region for a given $\beta$, and the shear becomes larger due to the larger shift of the magnetic axis and the consequent lowering of $\imath$ in the core region, so that the stability is improved.

The kink and ballooning stability properties are also improved when the current profiles become broader, if the pressure profile is kept fixed. A broader current profile moves the shear reversal point of the internal $\mathrm{l}$ further outward and makes the overall 


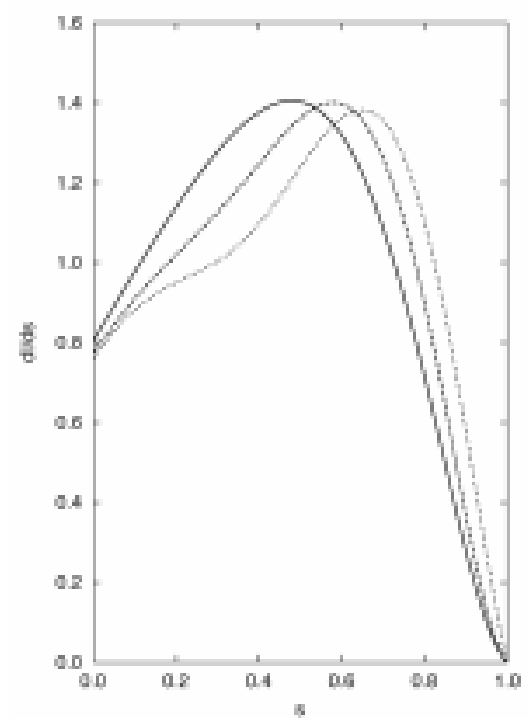

Fig. 4 Three hollow current profiles used for the stability analysis. J1: solid Line, J2: dashed line, J3: dotted line.

shear larger. Fig. 4 illustrates an example of three current profiles we use here for stability calculations. Fig. 5a shows the corresponding kink eignevalue as a function of $\beta$, and Fig. 5b shows the corresponding ballooning eigenvalue at $4 \% \beta$ as a function of $s$, all with the Aries pressure profile.
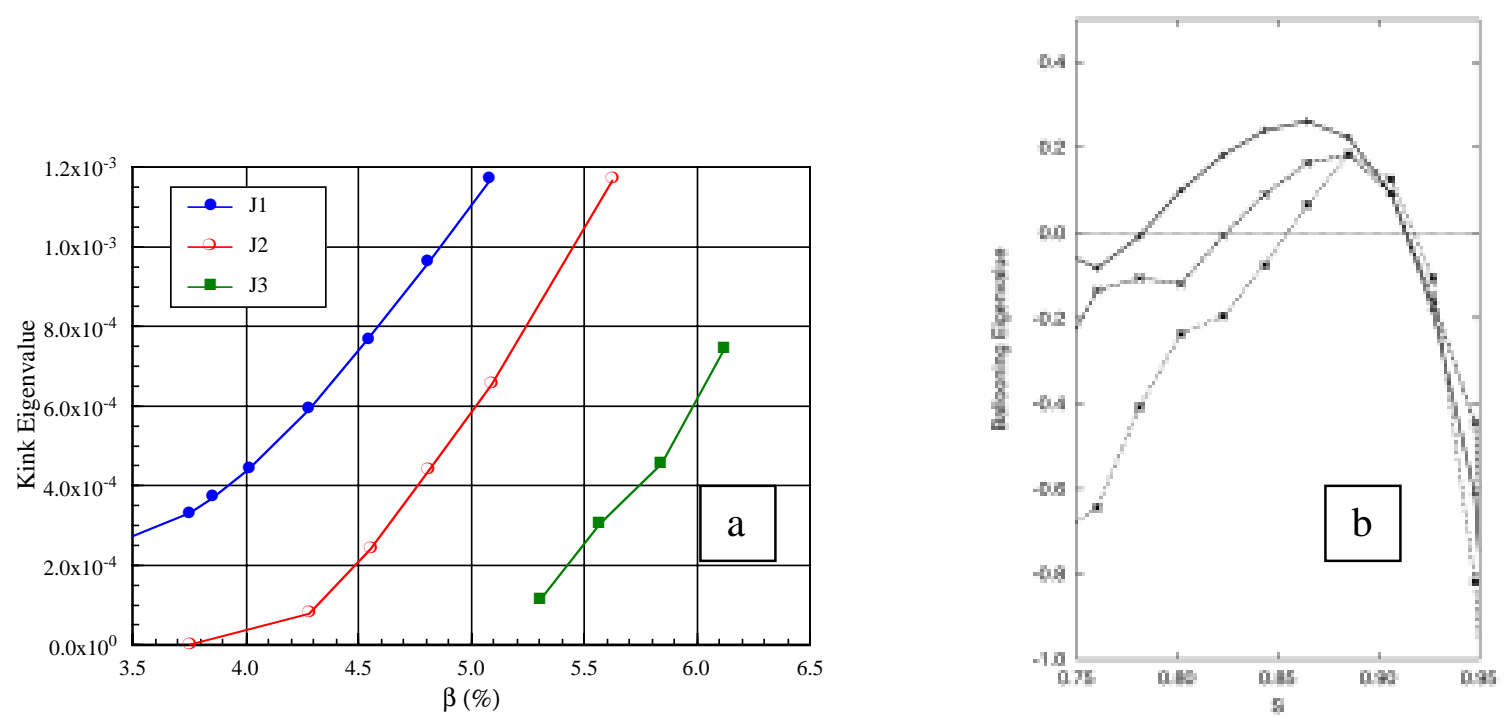

Fig. 5 (a) Kink eigenvalue versus $\beta$, and (b) ballooning eigenvalue at $4 \% \beta$ versus toroidal flux label s, showing stability improvement as current profiles become increasingly shifted outward. In (b), curve with solid line: J1, dashed line: J2, dotted line $\mathrm{J} 3$. 
Our configurations were typically optimized at $4 \% \beta$ and $200 \mathrm{kA}$ current for the size that fits into the space available for NCSX experiment. Moving away from the reference point will change the QA that has been optimized. Fig. 6a shows the $\chi^{2}$ for the three current profiles at $4 \% \beta$ and Fig. $6 \mathrm{~b}$ shows the $\chi^{2}$ for three $\beta$ s using J2 in Fig. 4. It is seen that QA is less sensitive to profile changes than to the changes in $\beta$ (and to the changes in the magnitude of current as well). The higher the pressure is, the less QA the configuration becomes.

When the current profile is derived from the bootstrap effects, the MHD stability favors a broader pressure profile since the resulting broader current profile has stronger stabilizing effects on the kinks and ballooning that more than compensate for the destabilizing effects caused by the broadening of the pressure profile.
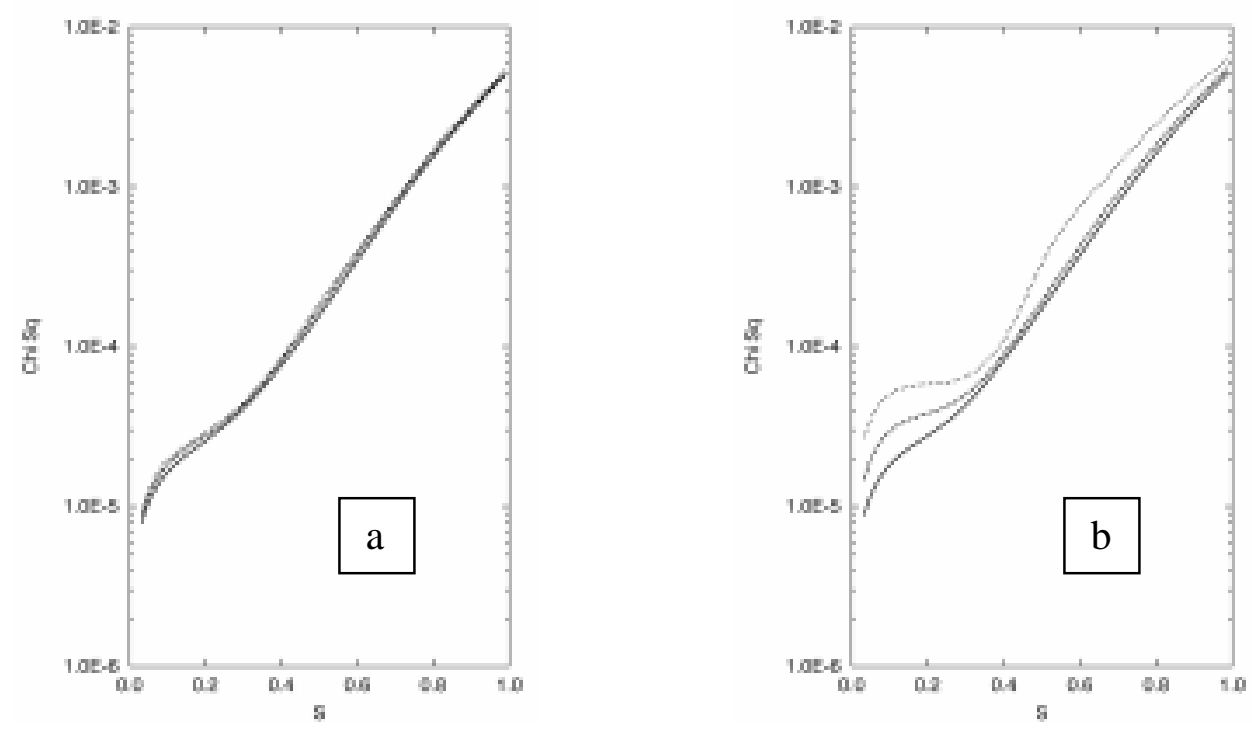

Fig. $6 \chi^{2}$ of non-axisymmetric magnetic field spectrum: (a) for three different current profiles given in Fig. 4 at $4 \% \beta$, (b) for $\beta=4 \%$ (solid), $5 \%$ (dashed) and $6 \%$ (dotted) with $\mathrm{J} 2$ current profile.

\section{EDGE ROTATIONAL TRANSFORM AND KINK STABILITY}

In [2], a stability diagram is given for the external kink modes for QAS3_C82. It is shown that as the pressure or current is increased, the configuration becomes less stable. A more careful study, however, shows that the kink growth rate decreases as the edge 1 approaches 0.5. A typical result is given in Fig. 7, where 1 is controlled by the amount of plasma current. The edge shear remains approximately the same. A slightly different configuration is used here to generate the kink eigenvalues; the coil current density was added as one additional parameter for optimization. As in QAS3_C82, the configuration 
is optimized at $200 \mathrm{kA}$ current with an edge $\mathrm{l}=0.466$. As the current is increased above the point of optimization, the kink growth rate initially increases, but it precipitates to a stable level as the current is further increased to the point where the edge $l$ is about 0.5 . Once the edge 1 exceeds 0.5 , the $m=2, n=1$ mode moves into the plasma, resulting in the rapid increase in the growth rate.

The above observation suggests that if one were to optimize this configuration at a current level corresponding to $\mathrm{l}=0.482$ in Fig. 7 , one could find a configuration that is more robust to kink modes as the current changes, as long as the edge 1 remains below 0.5. A new configuration, called QAS3_C99, based on this idea is shown in Fig. 8. Calculations indeed showed that the new configuration is kink stable to all plasma currents $<240 \mathrm{kA}$.

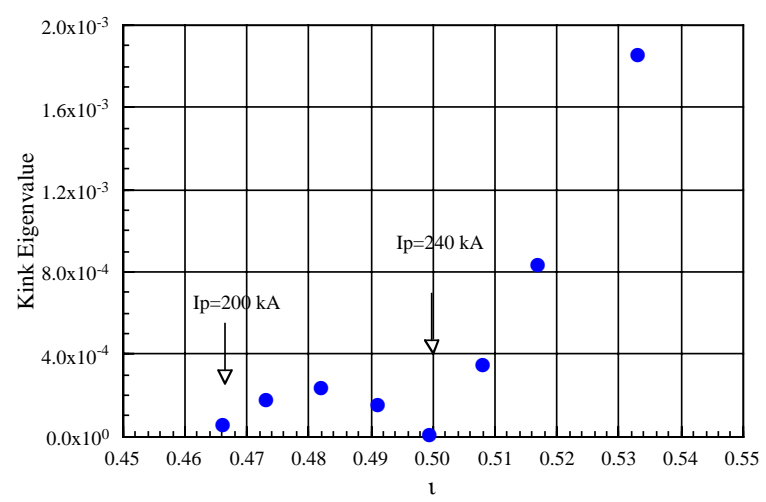

Fig. 7 Kink eigenvalue versus plasma edge rotational transform, $\mathrm{l}$, showing that the kink modes become stable as 1 approaches 0.5 .

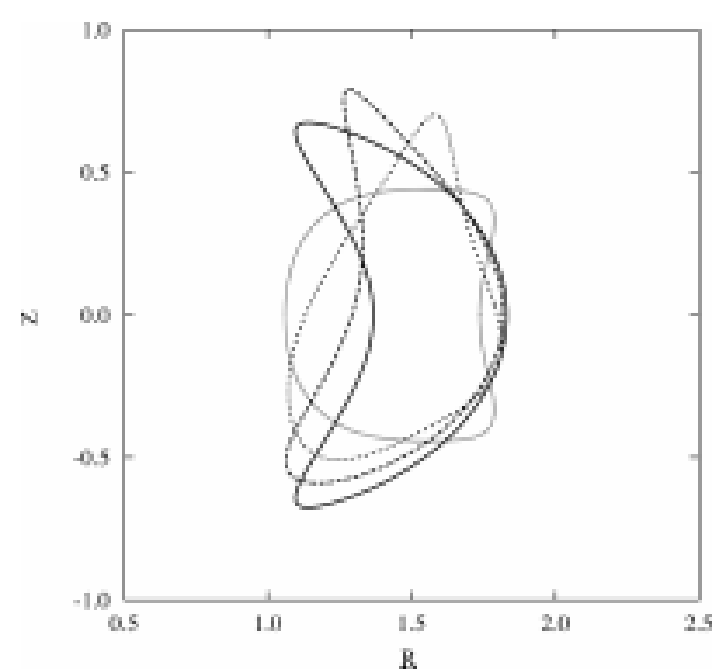

Fig. 8 Cross sections of QAS3_C99.

\section{SUMMARY AND CONCLUSIONS}

We have obtained quasi-axisymmetric configurations stable to ideal external kink, ballooning and vertical modes for NCSX. The results of studying their physics properties are discussed in this paper, in particular, for configuration QAS3_C82. The kink stability typical of our configuration favors an outboard indentation at the half-period cross section, the shaping of which involves detailed refinement requiring boundary modes of higher poloidal numbers (for example, $m=4$ ). On the other hand, significant QA is achieved in a few low order modes that effectively cancel out the large non-axisymmetric magnetic field components, particularly, $\mathrm{B}_{0,2}, \mathrm{~B}_{0,1}$, without compromising the kink 
stability. The study of the effects of profiles shows that MHD stability will be improved when the pressure profile becomes more peaked and the current profile gets broader. Thus, it is important to maintain certain current drive capability in experiments to allow modification of the current profile, particularly if the plasma current arises primary from the bootstrap effects. Finally, it is possible to optimize a configuration that is kink stable to all current levels for which the edge 1 is less than 0.5 .

\section{ACKNOWLEDGMENTS}

The authors wish to thank Drs. S. Hirshman and A. Cooper for help using VMEC, Terpsichore and VVBAL codes. This work was supported by the Department of Energy contract No. DE-AC02_76_CHO-3073.

\section{REFERENCES:}

[1] A. Reiman, L.P. Ku, D. Monticello, et al., Plasma Physics and Controlled Nuclear Fusion Research, 1998 (International Atomic Energy Agency, Vienna, 1999), paper IAEA-CN-69/ICP/06.

[2] A. Reiman, G. Fu, S. P. Hirshman, et al., in Proceedings of the $26^{\text {th }}$ European Physical society Conference on Controlled Fusion and Plasma Physics Research, Maastricht, the Netherlands, June 14-18, 1999, (European Physical Society, Petit-Lancy, 1999).

[3] S. C. Jardin, C. E. Kessel, C. G. Bathke et al., Fusion Engr. and Design, 38, 27 (1997).

[4] M. H. Redi, C. Nuehrenberg, W. A. Cooper et al., in Proceedings of the $26^{\text {th }}$ European Physical society Conference on Controlled Fusion and Plasma Physics Research, Maastricht, the Netherlands, June 14-18, 1999, (European Physical Society, PetitLancy, 1999).

[5] G. Fu, L. P. Ku and A. Cooper, This conference.

[6] P. Garabedian and L. P. Ku, Phys. of Plasmas, 6(3), 645 (1999).

[7] S. P. Hirshman, W. I. van Rij, and P. Merkel, Comput. Phys. Commun. 43, 143 (1986).

[8] D. V. Anderson, A. Cooper, U. Schwenn and R. Gruber, in Proc. of the Joint Varenna-Lausanne International Workshop on Theory of Fusion Plasmas, (Editrice Compositori, Bologna, 1988) p 93. 
[9] W. A. Cooper, Plasma Phys. Controlled Fusion 34, 1011 (1992). 